\title{
Analytical Chemistry and Power Engineering
}

DOI: $10.1134 / \mathrm{S} 1061934811010187$

Energy production, accumulation, delivery, and savings are the major scientific, engineering, and economic problems, no less in Russia. Chemical analysis and control play an important role in solving these problems.

For atomic and thermal power plants, the use of pure water is essential, because of which its analysis acquires major importance, primarily in terms of controlling the concentration of inorganic impurities (ion chromatography is highly convenient for this purpose). The gas industry is interested in efficient methods for detecting gas leakages and for chemical analysis, to say nothing of controlling gas composition. The petroleum industry requires an even higher extent of rational control; it is notable that industrial gas chromatographs are routine monitoring devices in this field. Determination of aromatic compounds and sulfur, sometimes vanadium, nickel, and other elements is only one constituent of the analytical control of oil and petroleum products. In motor fuels, it is necessary to control the presence of lead, iron, and water. In industrial oil delivery, it is desirable to control the concentration of chlorides. Environmental analytical control is necessary where oil is produced and pro- cessed, as well as in the restoration of contaminated areas.

The study and increasing use of alternative energy sources are directly associated with the necessity of analytical methods. This is particularly true for the production of biodiesel fuels and ethanol or dimethyl ether from various plant materials. The rational use of chemical processes in this case requires the determination of compositions at all stages of production and, certainly, the control of all products. The wide application of solar energy obtained using silicon wafers implies extended production of high-purity silicon, the composition of which is also controlled by chemical analysis methods. Analysis is also necessary in developing technology for the recovery of gas by-products of oil production, which nowadays are burned in torches. Analysts are making their own contribution to the development of new, more efficient chemical ion sources.

The multidisciplinary problem of power savings, in addition, includes control over engine wear, which can be achieved by analyzing cylinder oils.

Yu. A. Zolotov 